\title{
Experimental Results on Single Antenna Target Detection Using Time-Reversal Techniques
}

\author{
Ahmet G. Cepni* , Daniel D. Stancil, Ben Henty, Yi Jiang, Yuanwei Jin, \\ Jose M.F. Moura, Jian-Gang Zhu \\ Department of Electrical and Computer Engineering, \\ Carnegie Mellon University, Pittsburgh, PA 15213-3890, USA
}

\begin{abstract}
In this paper, we describe a single antenna time-reversal based detection scheme that can be used to enhance the performance of a radar system in a multipath rich scattering environment. Using time-reversal, the response from a cluttered medium is first nulled. When the target enters into the medium, the electromagnetic energy focuses around the target so that a stronger echo is obtained. The experimental results show that depending on the amount of clutter in the medium, the performance of a radar detection scheme can be improved up to $4.8 \mathrm{~dB}$ using time-reversal methods with a single antenna.
\end{abstract}

\section{INTRODUCTION}

Time-reversal is a novel method to utilize the multipath components in a cluttered environment for super-resolution focusing. The conventional thought about the adverse effects of multipath on communication systems has been changing based on recent findings showing how to use multipath components to create independent paths between transmitter and receiver. Time-reversal schemes are one of these recent techniques which can convert traditionally hostile multipath into performance-boosting elements in a two-way communication system as well as radar target detection systems in a cluttered channel. In a radar system, the matched filter deteriorates as the complexity of the medium is increased. On the contrary, time-reversal has better performance as the scattering environment gets more complicated. Time-reversal detection schemes can be implemented using a single antenna as well as multiple antennas. The timereversal adaptive interference cancellation (TRAIC) algorithm utilizes multiple antennas to detect the presence of a target in a highly cluttered channel [1],[2]. TRAIC relies on clutter nulling. By using time-reversal, the response from the cluttered medium is first nulled. When the target enters into the medium, the electromagnetic energy focuses around the target so that a stronger echo is obtained. The experimental results show that using the TRAIC algorithm, we can improve the signal-to-noise ratio of the target return echo compared to conventional change-detection radar. The TRAIC algorithm was also implemented using a single antenna [3]. Single antenna target detection using various timereversal algorithms and analytical expressions have been presented in [4]. In this paper, we will present experimental results of single antenna time-reversal techniques and compare the results with conventional change detection. 


\section{SINGLE ANTENNA TIME-REVERSAL DETECTION}

Time-reversal is equivalent to phase-conjugation in the frequency domain where the power detector analysis will be carried out. In the absence of the target, the clutter channel frequency response is given by $H_{c}(f)$. This is the background channel response. When the target enters into the medium, the channel frequency response becomes $H_{c t}(f)$. This can be decomposed into a component due to clutter $\left(H_{c}(f)\right)$ and a component due to the target $\left(H_{t}(f)\right)$. The target component also includes all higher order scattering from the clutter to the target. In the following, we assume that we can compute $H_{t}(f)$ by nulling the clutter and work only with the response from the target. The channel is assumed to be stationary, i.e. the clutter does not move. The forward and time-reversal channel responses can be written as

$$
\begin{gathered}
Y(f)=H_{t}(f)+N_{1}(f) \\
Z(f)=k Y^{*}(f) H_{t}(f)+N_{2}(f)
\end{gathered}
$$

where $N_{1}(f)$ and $N_{2}(f)$ are additive white noise at the receive ports and $k$ is a back-propagation constant that is used to make sure that the transmitted power is the same in each case. In the above equations $Y(f)$ is the received waveform due to change detection and $Z(f)$ is the received waveform after time-reversal. The power of the time-reversal based detector can be calculated as

$$
P_{T R}=\int|Z(f)|^{2} d f=\int\left|k Y^{*}(f) H_{t}(f)+N_{2}(f)\right|^{2} d f
$$

Similarly the power for a change-detection scheme is

$$
P_{C D}=\int|Y(f)|^{2} d f=\int\left|H_{t}(f)+N_{1}(f)\right|^{2} d f
$$

With a wide-band power detector (like a diode detector), the total power in the passband is measured in the presence of a target in the medium. In the absence of the target in the medium, the total noise power of the wide-band detector is measured, which increases proportionally to the total bandwidth. Using a 2 $\mathrm{GHz}$ bandwidth between $4 \mathrm{GHz}$ and $6 \mathrm{GHz}$, we have tested the performance of a single-carrier power detector in three scattering environments: no multipath, moderate multipath, and extreme multipath. In the first case, there are no scattering objects in the medium. A $2.4 \mathrm{~m}$ long copper pipe with a diameter of 1.27 $\mathrm{cm}$ is the only target in front of the C-band (4-6 GHz) horn antennas (Fig. 1a). The second case has moderate multipath components in the presence of $58 \mathrm{di}$ electric rods as the scattering channel and the same copper pipe target (Fig. 2a). The dielectric constant of the rods is 3.7 and the diameter is $3.2 \mathrm{~cm}$. The last case is the extreme multipath case, where a $5 \mathrm{~cm}$ long and $0.3 \mathrm{~cm}$ thick metal target is placed inside a $3 \mathrm{~m}$ long cylindrical duct with metal caps on both ends. The resonant duct cavity produces extensive echos due to the target (Fig. 3a). 

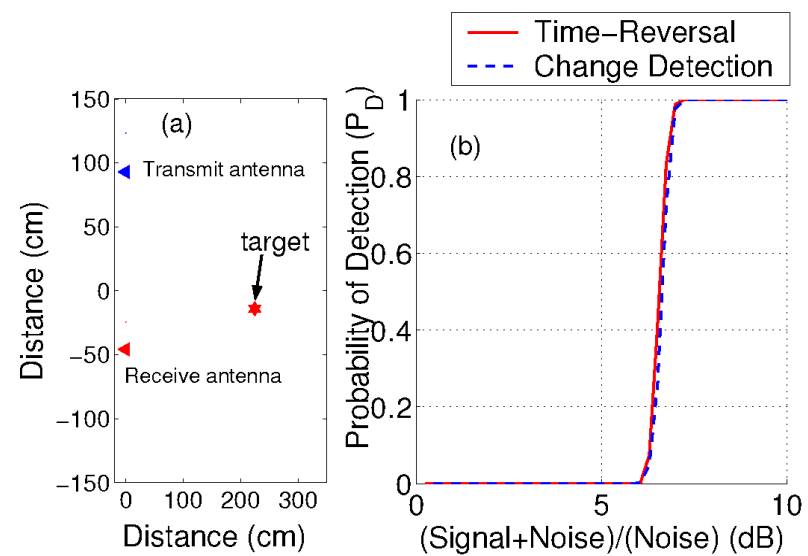

Fig. 1. The performance of time-reversal based detection and change detection in the presence of single target in the medium. No scattering objects. False Alarm Rate is $1 \%$.
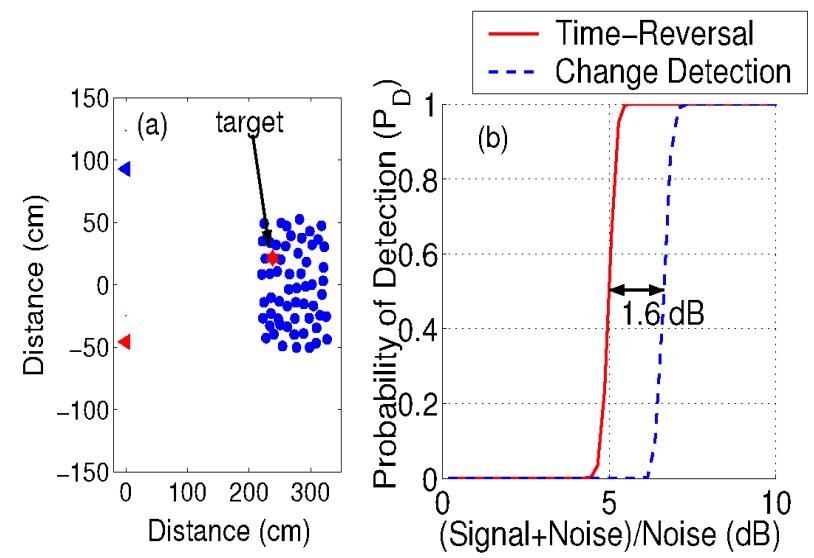

Fig. 2. The performance of time-reversal based detection and change detection in the presence of 58 scattering objects in the medium. The target is a copper pipe. False Alarm Rate is $1 \%$.

The detection performances of these three cases have been computed and compared with change detection results. There is no gain of time-reversal system over change detection in the first channel where there are no scattering objects in the medium (Fig. 1b). Fig. 2b compares the performance of time-reversal detection to change detection in the moderate multipath channel. The SNR requirement of change detection stays the same as the no multipath case, while the time-reversal curve shifted to the left by $1.6 \mathrm{~dB}$. Time-reversal thus provides a 1.6 $\mathrm{dB}$ improvement over change detection in this environment. In the extreme multipath case, the time-reversal curve is shifted even further to the left (Fig. 3b). The resulting performance improvement is $4.8 \mathrm{~dB}$ over change detection. 

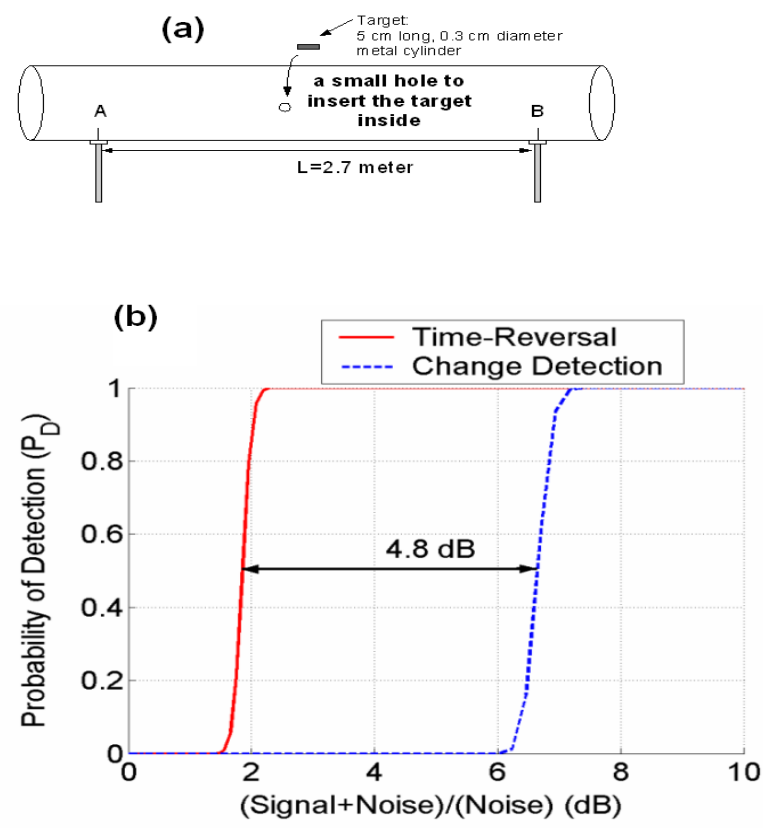

Fig. 3. The performance of time-reversal based detection and change detection in a multipath rich duct channel. The target is a $5 \mathrm{~cm}$ long $0.3 \mathrm{~cm}$ diameter metal cylinder. False Alarm Rate is $1 \%$.

\section{CONCLUSIONS}

We have shown that time-reversal methods can be used to design detection schemes that can work better in a cluttered medium. Single antenna time-reversal experiments have shown that the gain of the time-reversal based detection scheme is related to channel clutter. The more scattering the channel provides the better is the time-reversal gain. We have observed a $1.6 \mathrm{~dB}$ improvement in SNR in a laboratory environment where we have used 58 dielectric rods as scatterers and a copper pipe as the target. In a multipath rich duct channel, the time-reversal based detection scheme resulted in $4.8 \mathrm{~dB}$ improvement over change detection.

\section{REFERENCES}

[1] J.Zhu, Y. Jiang, D.D. Stancil, and J. Moura, "A Novel Time Reversal Method for Target Detection in Cluttered Media," IEEE Antennas and Propagation Society International Symposium, vol. 4B, pp. 135-138, Washington, DC, July'05.

[2] A.G. Cepni, D.D. Stancil, J.Zhu, Y. Jiang, "Experimental Results on Target Detection in Cluttered Medium using Electromagnetic Time-Reversal Techniques," IEEE Antennas and Propagation Society International Symposium, vol. 4B, pp. 139-142, Washington, DC, July'05.

[3] J. Moura, Y.W. Jin, D. Stancil, J.G. Zhu, A.G.Cepni, Y. Jiang, B. Henty, "Single Antenna Time Reversal Adaptive Interference Cancellation," IEEE International Conference on Acoustics, Speech, and Signal Processing, (ICASSP), Volume 4, pp. 1121-1124, March 2005.

[4] J. Moura, Y.W. Jin, "Detection by Time Reversal: Single Antenna," submitted to Journal of Acoustics, 2005. 